SCIENTIFIC REPORT

\title{
Effect of hypertension on foveolar choroidal haemodynamics
}

\author{
R M Niknam, L S Schocket, T Metelitsina, J C DuPont, J E Grunwald
}

Br J Ophthalmol 2004;88:1263-1265. doi: 10.1136/bjo.2003.038471

Aims: To assess the effect of systemic hypertension on the choroidal circulation in subjects with a normal eye examination.

Methods: Laser Doppler flowmetry was used to determine relative choroidal blood velocity $\left(\mathrm{Ch}_{\text {vel }}\right)$, volume $\left(\mathrm{Ch}_{\text {vol }}\right)$, and flow ( $\mathrm{Ch}_{\text {flow }}$ ) in the centre of the fovea. Measurements were obtained in 15 eyes of 15 subjects with systemic hypertension. The mean average duration of hypertension was 11 (SD 6) years. Findings obtained in these patients were compared with those of 15 eyes of 15 age matched healthy controls. All subjects had normal eye examinations.

Results: No significant differences in $\mathrm{Ch}_{\text {vel }}, \mathrm{Ch}_{\text {vol }}$, and $\mathrm{Ch}_{\text {flow, }}$ were found between the subjects with and without systemic hypertension despite a $17 \%$ higher perfusion pressure (PP) in patients with hypertension. No significant correlation was found between mean arterial pressure or PP and the choroidal circulatory parameters. It was estimated that there is a $90 \%$ power to detect a $35 \%$ difference in $\mathrm{Ch}_{\text {flow }}$ between the two groups.

Conclusion: Systemic hypertension does not seem to have a large effect on the choroidal circulation in hypertensive patients that are controlled by antihypertensive therapy. Further studies are needed to elucidate whether systemic hypertension has an effect on the choroidal circulation in patients with ocular diseases.

$\mathrm{S}$ ystemic hypertension is defined as persistently elevated arterial pressure that induces vascular constriction and sclerosis of both arteries and veins throughout the body. Systemic hypertension is a known risk factor for ophthalmic diseases such as diabetes, vascular occlusions, ocular ischaemic syndrome, glaucoma, and age related macular degeneration (AMD). ${ }^{1-4}$

The choroidal vasculature may be susceptible to the effects of hypertension because of its unique architecture. The choroidal arteries run a short course with relatively few branches and supply the choriocapillaries at right angles, thus systemic blood pressure is transmitted more directly to the choriocapillaries. ${ }^{5}$

The effect of systemic hypertension on ocular haemodynamics has been investigated in various ocular disease states. Grunwald et al reported that glaucoma patients with systemic hypertension had higher optic nerve blood flow than glaucoma patients without hypertension. ${ }^{6}$ Esgin and associates $^{7}$ measured pulsatile ocular blood flow, their results suggested that diabetics with systemic hypertension had an increased pulsatile ocular blood flow compared to age matched controls without systemic hypertension.

In addition, choroidal blood flow has been shown to be decreased in $\mathrm{AMD}^{8}$ Before an investigation of the effect of systemic hypertension on the choroidal circulation in AMD and other pathological states, it is important to ascertain whether systemic hypertension may affect choroidal flow in the normal eye. The goal of our study was to assess the effect of systemic hypertension on the choroidal circulation in such eyes.

\section{METHODS}

The study population included 15 subjects with systemic hypertension and 15 age matched healthy controls that were recruited at the Scheie Eye Institute, University of Pennsylvania. After a detailed explanation of the experimental procedures to be performed in this investigation, all subjects gave informed consent and were asked to sign a consent form approved by the institutional review board.

Eligibility criteria for both groups included no history of progressive ocular or retinal disease, a normal ophthalmic examination, Snellen visual acuity of 20/40 or better, and the ability to fixate on the laser beam.

All subjects had deep anterior chambers. Pupillary dilatation was achieved with $1 \%$ tropicamide (Alcon, Fort Worth, TX, USA) and $2.5 \%$ phenylephrine hydrochloride (Sanofi Winthrop, NY, USA). Ages ranged from 38 to 71 years (mean 54 (SD 11) years) for the hypertensive subjects, and from 34 to 76 (53 (14) years) for the control group. Twelve hypertensive patients were receiving antihypertensive medication and three patients were not receiving such medication.

Measurements of relative foveolar choroidal blood velocity $\left(\mathrm{Ch}_{\mathrm{vel}}\right)$, volume $\left(\mathrm{Ch}_{\mathrm{vol}}\right)$, and flow $\left(\mathrm{Ch}_{\mathrm{flow}}\right)$ were obtained using laser Doppler flowmetry (Oculix instrument). $\mathrm{Ch}_{\mathrm{vel}}$ represents the speed of erythrocytes, $\mathrm{Ch}_{\mathrm{vol}}$ is proportional to the number of erythrocytes present at the measured site, and $\mathrm{Ch}_{\text {flow }}$ is calculated by the instrument from $\mathrm{Ch}_{\mathrm{vel}}$ and $\mathrm{Ch}_{\mathrm{vol}}$. A detailed description of this technique has been published previously. ${ }^{9}$

A $20 \mu \mathrm{W}$ diode laser beam $(670 \mathrm{~nm})$ with a diameter of $200 \mu \mathrm{m}$ was delivered through a fundus camera (model TRC; Topcon, Tokyo, Japan). Subjects were asked to fixate on the probing laser beam. Proper fixation was confirmed by direct observation of the foveola through the fundus camera. This method allows for determination of choriocapillary flow as described by Riva et al. ${ }^{9}$

One eye chosen at random by the flip of a coin was studied in each subject. Three separate 30 second measurements of the choroidal circulation were obtained. A trained observer, masked to all attributes of the subjects, analysed the data using a NeXT computer (NeXT Computer, Inc, Redwood City, CA, USA) with software (NeXT Software, Inc, Redwood City, CA, USA) specifically designed for the analysis of Doppler signals from ocular tissues. ${ }^{10}$ Only portions of the recordings

Abbreviations: $A M D$, age related macular degeneration; $\mathrm{Ch}_{\text {vel, }}$ choroidal blood velocity; $\mathrm{Ch}_{\text {vol, }}$, choroidal blood volume; Chflow, choroidal blood flow; DBP, diastolic blood pressure; MBP, mean blood pressure; PP, perfusion pressure; SBP, systolic blood pressure 
Table 1 Characteristics of control subjects and hypertensive subjects (mean (SD))

\begin{tabular}{lllll}
\hline & Controls & Hypertension & \% Difference & p Value* \\
\hline Age (years) & $53(14)$ & $54(11)$ & 2 & 0.7 \\
IOP (mm Hg) & $15(2)$ & $15(3)$ & 0 & 0.72 \\
PP (mm Hg) & $46(7)$ & $54(7)$ & 17 & 0.008 \\
SBP (mm Hg) & $128(11)$ & $141(15)$ & 10 & 0.008 \\
DBP (mm Hg) & $74(10)$ & $84(11)$ & 14 & 0.012 \\
\hline *Non-paired, two tailed, Student's t test. & & &
\end{tabular}

that showed stable measurements were chosen for analysis and the results were averaged.

Upon completion of the blood flow measurements, heart rate, brachial artery systolic blood pressure (SBP), and diastolic blood pressure (DBP) were obtained with an automated sphygmomanometer. Mean blood pressure (MBP) was defined as:

$$
\text { DBP }+1 / 3(\text { SBP }- \text { DBP })
$$

The perfusion pressure (PP) was defined as:

$$
2 / 3 \mathrm{MBP}-\mathrm{IOP} \text {. }
$$

Results were compared using non-paired Student's $t$ test and probability values smaller than 0.05 were considered statistically significant.

\section{RESULTS}

There were no statistically significant differences in age and IOP between hypertensive patients and normal controls (table 1). In comparison with normal controls, however, hypertensive subjects had average SBP, DBP, and PP that were significantly higher than the normal subjects by $10 \%$, $14 \%$, and $17 \%$, respectively.

In comparison with normal controls, average $\mathrm{Ch}_{\mathrm{vel}}$ was $2.4 \%$ higher in the hypertensive subjects (non-paired Student's $t$ test; $\mathrm{p}=0.50$ ), average $\mathrm{Ch}_{\mathrm{vol}}$ was $13.9 \%$ lower in the hypertensive subjects (non-paired Student's $t$ test $\mathrm{p}=0.13)$, and average $\mathrm{Ch}_{\text {flow }}$ was $8 \%$ lower in the hypertensive subjects (non-paired Student's $t$ test $\mathrm{p}=0.60$; table 2). These differences between the subjects with and without systemic hypertension, however, were not statistically significant. $\mathrm{Ch}_{\text {flow }}$ measurements in normal subject and hypertensive patient are shown in figure 1 .

When hypertensive and control subjects were analysed together, a significant correlation between age and $\mathrm{Ch}_{\mathrm{vol}}$ $(r=-0.370 ; \mathrm{p}=0.044)$, and age and $\mathrm{Ch}_{\mathrm{flow}}(r=-0.388$; $\mathrm{p}=0.034)$ was observed. No significant correlation was detected, however, between age and $\mathrm{Ch}_{\mathrm{vel}}(r=0.208$; $\mathrm{p}=0.271)$. There was also no significant correlation between SBP, DBP, or PP and any of the circulatory parameters.

Because most of the hypertensive patients were on antihypertensive therapy, we examined whether these medications had an effect on circulatory parameters. There was no statistically significant difference in average $\mathrm{Ch}_{\text {flow }}$ between the 12 patients who were on antihypertensive medications (12.6 (4.8) arbitrary units, AU) and the three hypertensive patients who were not receiving antihypertensive medication (10.2 (2.3) AU; p = 0.25).

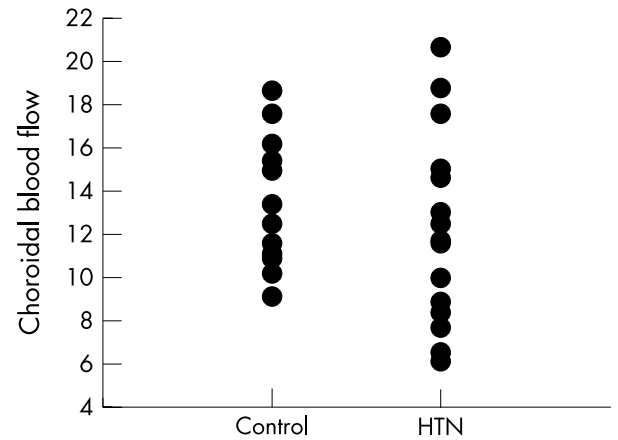

Figure 1 Relative foveolar choroidal blood flow in arbitrary units (AU) for control and hypertensive (HTN) subjects. There is no statistically significant difference in average choroidal blood flow between both groups (non-paired Student's $t$ test; $p=0.6$ ).

Because we did not detect any effect of hypertension on $\mathrm{Ch}_{\text {flow }}$ we have estimated that we have a $90 \%$ power to detect a $35 \%$ difference in $\mathrm{Ch}_{\text {flow }}$ between the two groups.

\section{DISCUSSION}

Although hypertensive choroidopathy and its clinical effects on the appearance of the fundus have been described, ${ }^{5}$ the effects of hypertension on the choroid before the appearance of clinically detectable fundus abnormalities have not been studied. This information is important, however, because the choroidal circulation is crucial for the maintenance of normal retinal function as it provides nutrients and removes waste products from the outer half of the retina and is the main source of oxygen and nutrients to the avascular fovea.

Retinal blood vessels regulate blood flow in response to blood pressure, intraocular pressure, and the content of oxygen in the blood. Choroidal vessels have been shown in rabbits to have the capability to autoregulate in response to changes in blood pressure and intraocular pressure. ${ }^{11}{ }^{12}$ Studies in humans have also shown that choroidal blood flow is maintained despite an increase in perfusion pressure, suggesting that in humans the choroidal vasculature may autoregulate. ${ }^{13-15}$

This ability of the choroidal circulation to autoregulate may explain why we found no significant differences in $\mathrm{Ch}_{\mathrm{vel}}$, $\mathrm{Ch}_{\mathrm{vol}}$, and $\mathrm{Ch}_{\text {flow, }}$ between the subjects with and without systemic hypertension in spite of the fact that PP was 17\% higher in the hypertensive subjects (Student's $t$ test; $\mathrm{p}=0.008$ )

Table 2 Average choroidal circulatory parameters in arbitrary units (AU) (mean (SD))

\begin{tabular}{lllll}
\hline & Controls & Hypertension & \% Difference & p Value* \\
\hline Velocity (AU) & $0.41(0.08)$ & $0.42(0.07)$ & 2.4 & 0.5 \\
Volume (AU) & $0.36(0.07)$ & $0.31(0.09)$ & -13.9 & 0.1 \\
Flow (AU) & $13(3)$ & $12(4)$ & -8 & 0.6 \\
\hline *Non-paired, two tailed Student's t test & & & \\
\hline
\end{tabular}


We examined whether hypertensive patients taking antihypertensive medications $(n=12)$ had different choroidal circulatory parameters from those who did not take such medications $(\mathrm{n}=3)$. We did not find any statistically significant differences between the two groups. However, a word of caution should be mentioned with regard to this statement since the number of patients included in our study is small and this does not allow us to reach any strong conclusions.

Several studies on the effects of antihypertensive therapy on the choroidal circulation have been reported. A study of the effect of the calcium channel antagonist felodipine has suggested no significant effect on the choroidal blood flow in spite of a significant decrease in blood pressure. ${ }^{16}$ Others have used the laser speckle method in rats and have suggested that nilvadipine and pranidipine increase choroidal blood velocity. ${ }^{17}{ }^{18}$

When we analysed all the patients together, we found an inverse correlation between age and $\mathrm{Ch}_{\mathrm{vol}}$, and age and $\mathrm{Ch}_{\text {flow }}$. This is consistent with the report of Grunwald et al ${ }^{19}$ that showed that $\mathrm{Ch}_{\mathrm{vol}}$, and $\mathrm{Ch}_{\text {flow }}$ decrease with age. Because aging has an effect on $\mathrm{Ch}_{\mathrm{vol}}$, and $\mathrm{Ch}_{\text {flow, }}$ it is important to note that both groups were well age matched.

Systemic hypertension does not seem to have a large effect on the choroidal circulation in hypertensive patients that are controlled by antihypertensive therapy or in patients with a recent diagnosis of hypertension that don't have clinical evidence of hypertensive retinopathy. It may be that within the range of blood pressure that we examined, the choroidal circulation is maintaining the blood flow by an autoregulatory mechanism.

Further studies are needed to examine whether systemic hypertension and its treatment have an effect on the choroidal circulation in patients with ocular diseases.

\section{Authors' affiliations}

R M Niknam, L S Schocket, T Metelitsina, J C DuPont, J E Grunwald, Scheie Eye Institute, University of Pennsylvania, 51 North 39th Street, Philadelphia, PA 19104, USA

Correspondence to: Dr J E Grunwald, Scheie Eye Institute, University of Pennsylvania, 51 North 39th Street, Philadelphia, PA 19104, USA; juangrun@mail.med.upenn.edu
Accepted for publication 29 February 2004

\section{REFERENCES}

1 Epstein M, Sowers JR. Diabetes mellitus and hypertension (Review). Hypertension 1992; 19:403-18.

2 Messerli FH, Grossman E, Michalewicz L. Combination therapy and target organ protection in hypertension and diabetes mellitus. (Review) Am J Hypertens 1997; 10(pt 2):198S-201S.

3 Sperduto RD, Hillar R. Systemic hypertension and age related maculopathy in the Framingham Study. Arch Ophthalmol 1986;104:216-19.

4 Bischoff $\mathbf{P}$, Flower RW. High blood presssure in choroidal arteries as a possible pathogenetic mechanism in senile macular degeneration. Am J Ophthalmol 1983;96:398-9.

5 Tso MO, Jampol LM. Pathophysiology of hypertensive retinopathy. Ophthalmology 1982;89:1132-45.

6 Grunwald JE, Piltz J, Hariprasad SM, et al. Optic nerve blood flow in glaucoma: effect of systemic hypertension. Invest Ophthalmol Vis Sci 1998;39:2329-36.

7 Esgin $\mathrm{H}$, Alimgil ML, Erda S. The effect of systemic hypertension on pulsatile ocular blood flow in diabetic patients. Acta Ophthalmol Scand 2001;79:160-2.

8 Grunwald JE, Hariprasad SM, DuPont J, et al. Foveolar choroidal blood flow in age-related macular degeneration. Invest Ophthalmol Vis Sci 1998;39(No 2):385-90.

9 Riva CE, Cranstoun SD, Grunwald JE, et al. Choroidal blood flow in the foveal region of the human ocular fundus. Invest Ophthalmol Vis Sci 1994;35:4273-81.

10 Petrig BL, Riva CE. New continuous real-time analysis system for laser-doppler flowmetry and velocimetry in the ocular fundus using a digital signal processor. Wahsington, DC: Optical Society of America. Vision Science and its Application Technical Digest Series 1994;2:238-41.

11 Kiel JW, Shepherd AP. Autoregulation of choroidal blood flow in the rabbit. Invest Ophthalmlo Vis Sci 1992;33:2399-410.

12 Kiel JW. Modulation of choroidal autoregulation in the rabbit. Exp Eye Res 1999;69:413-29.

13 Riva CE, Titze $P$, Hero $M$, et al. Effect of acute decreases of perfusion pressure on choroidal blood flow in humans. Invest Ophthalmol Vis Sci 1997;381:752-60.

14 Riva CE, Titze $P$, Hero $M$, et al. Choroidal blood flow during isometric exercises. Invest Ophthalmol Vis Sci 1997;38:2338-43.

15 Robinson F, Riva CE, Grunwald JE, et al. Retinal blood flow autoregulation in response to an acute increase in blood pressure. Invest Ophthalmol Vis Sci 1986;27:722-6.

16 Schocket LS, Grunwald JE, DuPont J. Effect of oral felodipine on ocular circulation. Int Ophthalmol 1999;23:79-84.

17 Tomita K, Araie M, Tamaki Y, et al. Effects of nilvadipine a calcium antagonist, on rabbit ocular circulation in NTG subjects. Invest Ophthalmol Vis Sci 1999;40:1144-51.

18 Tamiki Y, Araie M, Tomita K, et al. Effects of pranidipine, a new calcium antagonist, on circulation in the choroid, retina, and optic nerve head. Curr Eye Res 1999;19:241-7.

19 Grunwald JE, Hariprasad SM, DuPont J. Effect of aging on foveolar choroidal circulation. Arch Ophthalmol 1998;116:150-4. 